\title{
Entrelacs
}

Cinéma et audiovisuel

14 | 2018

Marchés du film : évolutions, mutations et perspectives

\section{La disparition du marché du film en salles d'Afrique sud saharienne francophone (1960-2018)}

Claude Forest

\section{OpenEdition}

\section{Journals}

Édition électronique

URL : http://journals.openedition.org/entrelacs/4214

DOI : 10.4000 /entrelacs.4214

ISSN : 2261-5482

Éditeur

Éditions Téraèdre

Référence électronique

Claude Forest, «La disparition du marché du film en salles d'Afrique sud saharienne francophone (1960-2018) », Entrelacs [En ligne], 14 | 2018, mis en ligne le 17 décembre 2018, consulté le 03 mai 2019. URL : http://journals.openedition.org/entrelacs/4214 ; DOI : 10.4000/entrelacs.4214

Ce document a été généré automatiquement le 3 mai 2019.

Tous droits réservés 


\title{
La disparition du marché du film en salles d'Afrique sud saharienne francophone (1960-2018)
}

\author{
Claude Forest
}

1 Dans le combat pour l'autonomisation du cinéma en Afriques, très peu d'Africains francophones ont cherché à mener des travaux historiques ou économiques sur l'évolution de ce marché ${ }^{1}$, et encore moins d'Européens s'y sont intéressés ${ }^{2}$. Pourtant, plus d'un demi-siècle après, la profession de producteur de film n'a toujours pas émergé, les salles de cinéma ont quasiment disparu de toute l'aire, et la situation matérielle des professionnels de la filière est catastrophique dans l'ensemble de l'Afrique sud saharienne francophone $(\mathrm{ASF})^{3}$. Elle se trouve en revanche en ébullition en Afrique anglophone depuis longtemps, et pas seulement au Nigeria fortement médiatisé depuis un quart de siècle. Pour expliciter la disparition du marché cinématographique sur toute la zone, nous montrerons la responsabilité des cinéastes et des États africains, qu'a aggravé la forme de « l'aide » française.

2 Au niveau du cinéma, durant la colonisation, en ASF l'amont de la filière cinéma (production) était entièrement assuré par des métropolitains (Français, Belges), les stades intermédiaires (importation des films, distribution, programmation des salles) étant contrôlés par un faible nombre d'entreprises occidentales, essentiellement le duopole Secma et Comacico sur l'ancienne zone française. Seules les salles possédaient un statut plus mélangé, s'assimilant aux autres commerces, pouvant être gérées et/ ou possédées par des Métropolitains, des Nationaux voire quelques étrangers, Libanais notamment. Mais l'évolution du cinéma depuis les indépendances juridiques des anciennes colonies ne peut être déconnectée, ni de ses origines puis de l'évolution de la situation géopolitique, ni de la place de la filière au sein de fonctionnements socio-économiques et culturels beaucoup plus larges dans tous les pays concernés, ni se situer à l'extérieur des problématiques politiques autour des modes de développement des nations concernées. 
3 La lutte d'influence entre les deux grandes puissances de l'époque, les États-Unis et l'URSS, se jouera aussi en Afrique, notamment francophone et lusophone, impactant indirectement le cinéma. On ne saurait comprendre les postures et revendications des cinéastes africains durant les deux premières décennies post indépendances sans les replacer dans ce contexte de la Guerre froide, pas plus que la destruction totale de la filière cinéma après la chute du mur de Berlin, prélude à l'effondrement de l'empire soviétique et à l'estompement de ses jeux d'influence.

\section{L'Afrique francophone et son cinéma, victimes des jeux d'influence}

4 Pour des raisons géopolitiques - laisser la France gérer son pré-carré -, et commerciales - le très faible niveau de recettes cinéma sur la zone -, l'impact des États-Unis d'Amérique, premier exportateur mondial de films, s'est toujours avéré extrêmement marginal en ASF, même durant leur brève tentative de pénétrer ce marché au cours de la décennie 1970. En revanche leurs pays ont été influencés durant un quart de siècle dans leurs politiques, dont cinématographiques, par trois sources principales: l'URSS et certains de ses alliés, la recherche d'une voie autonome autour du panafricanisme, et la France et sa politique de coopération (que nous ne développerons pas ici) ${ }^{4}$.

\section{La tentative hégémonique des pays socialistes}

5 Après avoir initialement totalement ignoré les territoires africains, à partir de 1956 l'URSS chercha à exercer en Afrique une ascendance sur tous les fronts, idéologique (formation en Russie des cadres...), politique (encouragement aux indépendances...), économique (nationalisations...), culturelle et éducative (formation aux techniques cinématographiques à l'institut du cinéma de Moscou [VGIK], organisation de festivals...). Souhaitant en faire de nouvelles lignes de front de la guerre froide, en substituant la lutte contre les impérialismes à la lutte des classes, elle arrivera à étendre son attraction, s'appuyant également sur d'autres nations socialistes : Cuba, la République Démocratique Allemande, la Yougoslavie et la Tchécoslovaquie notamment. Par exemple, si la majorité des apprentis cinéastes d'ASF se formera en France, nombreux seront ceux qui passeront par le VGIK de Moscou, mais en ASF son influence directe sera limitée, et passera plutôt par d'autres pays "frères" du continent, l'un directement, la Tunisie, et l'autre indirectement, l'Algérie. Mais l'évidence de la tentative d'instrumentalisation de la part de l'URSS rebutera nombre de réalisateurs africains qui créeront en 1969 leur propre festival, le Fespaco ${ }^{5}$, qui se tient depuis tous les deux ans dans la capitale de l'actuel Burkina Faso, Ouagadougou, en alternance avec les Journées cinématographiques de Carthage en Tunisie, créées dès 1966.

\section{L'influence de l'Algérie et de Tunisiens}

6 Pendant longtemps, le jeune pouvoir algérien n'aura de cesse de s'opposer voire de continuer à combattre la France sur tous les terrains, notamment culturels et commerciaux. Très active politiquement et militairement contre les impérialismes, son orientation politique socialisante l'amènera à soutenir matériellement, voire à manipuler, tous les groupes pouvant s'opposer à la France, y compris dans le champ intellectuel et 
culturel, en poussant à leur radicalisation, quoiqu'apparaissant rarement au premier plan. Au niveau du cinéma l'enjeu économique était faible, mais l'aspect symbolique puissant, satisfaisant de surcroit la pression des cinéastes africains pour tenter de vivre de leur art, mettant en avant l'argument politico-culturel de la nécessité de décoloniser les regards et les écrans.

7 La théorisation de ce combat fut davantage le fait de Tunisiens, Férid Boughedir ${ }^{6}$ et Tahar Cheriaa $^{7}$, qui pousseront à l'intervention des États dans le cinéma, et notamment la nationalisation de sa distribution qui interviendra (dans la loi, mais pas dans les faits) dès janvier 1969 en Tunisie, l'Algérie instaurant en mai de la même année son monopole public national d'importation et de distribution des films. Si la nationalisation des salles, puis de la coproduction, n'avait rien modifié dans les relations internationales ni dans la structure de la filière, celle de la distribution provoquera de vives réactions. Les majors hollywoodiennes boycotteront l'Algérie jusqu'en 1974 en refusant de lui céder les droits d'exploitation de leurs titres, et elle aura dès lors le plus grand mal à s'alimenter en films, ceux en provenance des pays frères d'Europe de l'Est ne répondant que très partiellement aux attentes de la population. L'Egypte, dont la production de films représentait près des deux tiers de celle de tout le continent, n'assurera aucun soutien en alimentation de films, ni à ces pays du Maghreb, ni à ceux d'ASF lorsqu'ils se lanceront à leur tour dans un affrontement avec les industries du Nord. Dès lors, ce sera le début des difficultés pour les salles d'Afrique du Nord, d'abord en Algérie, par désaffection des spectateurs, jusqu'à leur disparition quasi complète par désintérêt ultérieur total de la part des pouvoirs publics, alors que ce pays en abritait le plus grand nombre au lendemain des indépendances.

Tableau 1 : Parc des salles en Afriques francophones : 1972 et 2018

(Classement par zone et nombre de salles décroissant en 1972)

\begin{tabular}{|l|l|l|l|}
\hline Pays & $\begin{array}{l}\text { Année } \\
\text { l'indépendance }\end{array}$ & $\begin{array}{l}\text { Salles en } \\
1972 \\
(16+35 \mathbf{~ m} / \mathbf{m})\end{array}$ & $\begin{array}{l}\text { Salles en 2018 } \\
(35 \mathrm{~m} / \mathrm{m}+\mathbf{D C P})\end{array}$ \\
\hline Afrique du Nord & & $250+250$ & $\sim 10$ \\
\hline Algérie & 1962 & $40+210$ & 60 \\
\hline Maroc & 1956 & $38+76$ & 18 \\
\hline Tunisie & 1957 & $(323)$ & $\begin{array}{l}(41, \\
\text { numérisées })\end{array}$ \\
\hline Afrique sud saharienne & & 70 & 8 \\
\hline Sénégal & 1960 & 48 & 5 \\
\hline Côte d'Ivoire & 1960 & 45 & 6 \\
\hline Rép. Dém. Congo & 1960 & 32 & 3 \\
\hline Cameroun & 1960 & & 30 \\
\hline
\end{tabular}




\begin{tabular}{|l|l|l|l|}
\hline Gabon & 1960 & 30 & 2 \\
\hline Guinée & 1958 & 28 & 2 \\
\hline Soudan/Mali & 1960 & 18 & 2 \\
\hline Rép. Populaire du Congo & 1960 & 13 & 3 \\
\hline $\begin{array}{l}\text { Haute-Volta/ Burkina } \\
\text { Faso }\end{array}$ & 1960 & 10 & 5 \\
\hline Niger & 1960 & 10 & 2 \\
\hline Togo & 1960 & 7 & 1 \\
\hline Tchad & 1960 & 6 & 1 \\
\hline Centrafrique & 1960 & 3 & 0 \\
\hline Dahomey/Bénin & 1960 & 3 & 1 \\
\hline
\end{tabular}

Sources : Unesco, UniFrance, Vieyra

Nota : il faut préciser que le recensement précis des salles est très délicat, nombre d'entre elles ne fonctionnant pas en permanence, ou certains lieux non spécialisés, mais équipés en vidéoprojecteur diffusant aléatoirement des films non déclarés, etc.

L'intensité des combats, politiques et/ou armés, contre les colonisateurs européens, et la cause commune des pays du continent africain pour leur indépendance, vont conduire les cinéastes et leurs alliés à se rapprocher, voire tenter de globaliser leur lutte, créant l'utopie d'une correspondance de situations entre le nord et le sud du Sahara, et donc de solutions que certains ont cru pouvoir considérer similaires si ce n'est communes. Or, pour le cinéma, en raison de différences structurelles historiques, culturelles, de taille des populations, de taux d'urbanisation, comme de richesse et de développement des pays, la situation des pays d'Afrique du Nord qui étaient plus développés économiquement, plus expérimentés dans la gestion de l'État, les rendaient, littéralement, incomparables avec ceux de l'ASF. Par exemple, à la date des indépendances, un seul pays comme l'Algérie comptait plus de salles de cinéma que les quatorze pays d'Afriques Occidentale et Equatoriale Françaises réunis! Dès lors, avoir voulu appliquer des solutions de même ordre pour réformer la filière cinéma au lendemain des indépendances partait d'a priori dogmatiques ou utopistes, pas d'une analyse réaliste de l'économie du secteur. Que trois fois moins de pays (le Maghreb), proposent deux fois et demie plus de salles, qui accueillent 4,5 fois plus de spectateurs avec un taux de fréquentation annuelle par habitant près de huit fois supérieur, en produisant près de huit fois plus de films, constituait un état de fait majeur qui aurait du interdire toute comparaison, et encore moins transposition de problématiques d'une zone à l'autre. Or, l'aveuglement idéologique conduira nombre d'intellectuels et cinéastes à penser que ce qui s'était appliqué pour le cinéma en Afrique du Nord pouvait pareillement s'appliquer au sud du Sahara, sans même attendre les résultats des expériences en cours. Cela constituera une mortelle erreur pour la filière, avant que les échecs des nationalisations des années $1970^{8}$ et le poids des réalités économiques suivies des pressions des institutions financières 
internationales, ne provoquent un mouvement inverse de privatisation les deux décennies suivantes.

\section{Illusions idéologiques du panafricanisme}

9 C'est donc à Alger, dans le cadre du Festival Panafricain de 1969, que la Fepaci (fédération panafricaine des cinéastes) sera conçue avant d'exister juridiquement l'année suivante à Tunis. C'est également à Alger que se radicalisera la mobilisation de ces mêmes cinéastes en faveur d'un combat anti-impérialiste et panafricaniste lors de son deuxième congrès, le 18 janvier 1975. Ce militantisme initial va la conduire à refuser tout cinéma commercial, à prôner un cinéma exclusivement tourné vers l'éducation et l'émancipation des peuples, à fabriquer l'utopie d'un affranchissement des contraintes économiques par simple volontarisme politique. Ce combat connaitra son acmé dans la Charte d'Alger adoptée à l'unanimité lors de ce congrès: très symptomatiquement, les mots de " publics », « spectateurs », « salles de cinéma », " distribution de films » n'apparaissent jamais. La seule indication économique assène que «la considération de rentabilité commerciale ne saurait être une norme de référence pour le cinéaste africain ». Tout est dit, et c'est précisément cette radicalité ignorante des contraintes de l'industrie du cinéma qui conduira en une décennie à la destruction complète de la distribution, puis de toute la filière sur l'ASF. Rejetant le cinéma "commercial » sans lequel il n'est point d'industrie cinématographique, méprisant les goûts de leurs publics qu'ils jugeaient seulement abêtis par l'offre de films occidentaux ${ }^{9}$, confondant leurs intérêts légitimes réaliser des films - avec ceux de leurs peuples, ignorants du fonctionnement du marché des salles, myopes au repli nationaliste qu'engageaient tous leurs dirigeants politiques, incapables de fédérer des forces qui nécessitaient d'être au moins sous-continentales, répétant une doxa marxisante qui voyait en la nationalisation la solution à tous les problèmes économiques, ils réussiront à pousser certains de leurs dirigeants politiques à une intervention brouillonne, non préparée et non coordonnée. Vouée à l'échec.

\section{Maladresse et désintérêt des États}

10 Si l'aveuglement idéologique peut s'expliquer au vu des rapports de force et expériences de la période, les erreurs d'analyse des cinéastes de la Fepaci ont été nombreuses, alors que d'aucuns les avaient déjà éclairés sur les préalables pour la réussite d'une autonomisation de la filière cinéma ${ }^{10}$. Tout d'abord, ils présupposaient que, au vu des expériences tunisienne et algérienne :

11 a) les situations d'Afrique du Nord étaient transposables sur tout le continent (supra), illusion à laquelle ont adhéré les cinéastes par croyance panafricaniste et enthousiasme anti-impérialiste ;

b) s'appuyant sur le leitmotiv de Cheriaa « celui qui tient la distribution tient le cinéma » 11, affirmation éminemment discutable, mais qui pouvait se comprendre au vu des expériences de son pays, elle nécessitait surtout pour réussir deux actions immédiates concomitantes qui ne seront pas mises en œuvre :

13 1) la première aurait été un immédiat contrôle des recettes des salles, que le duopole Secma-Comacico assurait rigoureusement en interne, mais qui, à ce jour encore, n'a été institué dans aucun pays d'ASF. En effet, au contraire des marchés ordinaires où la fraude 
et la corruption ne lèsent «que » l'État et ne pénalise pas la production, il n'en va pas de même pour le cinéma. Fraude ou malversation, lorsque la remontée de recettes des salles n'opère plus, la distribution étant rémunérée proportionnellement à ces recettes, cette activité ne devient plus rentable et les entreprises disparaissent, puis il en va ensuite de même pour la production, elle aussi rémunérée proportionnellement selon le même principe. Ce phénomène se produira au cours des années 1980, dans tous les pays d'ASF. Le mécanisme sera en partie voilé durant un temps car la France et certains organismes (type Organisation internationale de la francophonie) continueront durant longtemps à financer la production de films, mais sans aucun marché ni débouché national pour eux, dans une logique "d'aide ", qui profitera avant tout à l'image et à l'industrie françaises (la majorité des fonds alloués devant notamment être dépensée en France) ${ }^{12}$, favorisera quelques réalisateurs africains, produira indéniablement certains films jugés de qualité, mais empêchera de facto des industries techniques nationales (production, postproduction, studios, laboratoires...) d'émerger.

14 2) la seconde aurait été la conservation d'un marché d'une taille économiquement suffisante. Or, sur des territoires géographiquement treize fois supérieurs à la France, mais avec une population inférieure d'un tiers, et moins de salles cumulées qu'une seule ville comme Paris, la rentabilité, et donc la simple existence pérenne de distributeurs reposait sur un marché unifié et cohérent, tant en législation, fiscalité, circulation des copies, etc. Cette base ne sera jamais constituée, ni par l'initiative privée après la vente du duopole Secma-Comacico qui l'avait assurée jusqu'en 1974, ni par une volonté publique régionale. Dès lors, tous les marchés nationaux - avec souvent seulement 6 à 30 salles dans chaque pays - seront trop étroits pour pouvoir négocier des films en quantité suffisante, à des prix accessibles, ni les faire circuler entre territoires.

15 Accessoirement, il aurait également convenu de mettre des personnes compétentes, formées, et soucieuses de l'intérêt collectif, la multitude de fonctionnaires inexpérimentés et souvent peu scrupuleux qui seront affectés aux salles nationalisées ne l'étant pas sur la base de leur connaissance des métiers de l'exploitation. Cela aurait donc nécessité une préparation puis une intervention publique des États - pour laquelle les cinéastes militèrent beaucoup, et déplorèrent longtemps l'absence - tant aux niveaux réglementaire, régulateur que financier. Or, si un groupement d'États promit en 1970 une structure de distribution, elle ne verra le jour qu'en 1979 (le Consortium interafricain de distribution cinématographique - CIDC), mais un seul pays, le Niger, l'abonda financièrement, cinq autres en partie, le non-respect de tous les autres provoquant sa cessation d'activités dès 1985 après un fonctionnement incomplet et boiteux, qui ne sera jamais retenté ensuite. Sans remontée de recettes, plus de distributeurs, sans distributeur, pas de film, sans film, plus de salles, sans salle, plus de financement pour les films : l'exact inverse de ce qui était visé se produisit en deux décennies.

Au cours de cette période, ces cinéastes et politiques africains, combattant légitimement toute forme d'impérialisme et de volonté hégémonique étrangère, opèreront une confusion entre une domination économique indéniable de l'ancien duopole français qui perdurait encore une décennie après les indépendances, et ce qu'il en aurait été de sa volonté, à la fois d'abêtir les masses africaines par des offres de sous-produits occidentaux motivée par des considérations idéologiques d'une part, et de l'autre de ce qu'il en aurait été d'une très grande profitabilité du marché avec rapatriement en métropole de bénéfices fantasmés considérables ${ }^{13}$. Cette croyance, totalement erronée économiquement et qui ne reposait que sur des présupposés idéologiques, interdira 
d'analyser le véritable fonctionnement du duopole ${ }^{14}$. En effet, ces deux entreprises étaient intégrées verticalement, et, seul, le cumul des fonctions en assurait la rentabilité. Elles possédaient ou contrôlaient la moitié des salles de l'ASF, ce qui en faisait la partie visible de leur action (d'où leur nationalisation, à commencer par la Haute Volta en 1970). Par un montage juridique habile et complexe, elles assuraient également toutes les autres fonctions liées à la vie du film en salles, à commencer par la programmation de leurs salles, mais surtout celles de nombreuses autres sur la zone. Elles distribuaient également les films qui y circulaient, dont elles étaient seules à détenir les droits sur l'ensemble des territoires concernés, souvent pour une longue durée (cinq ans), puisqu'étant d'abord les importateurs de la quasi-totalité des titres proposés en ASF.

Importation, distribution, programmation, exploitation, et même achat et entretien du matériel technique (la Comacico était le représentant exclusif des robustes projecteurs 35 $\mathrm{m} / \mathrm{m}$ Cinemeccanica) leur permettaient ainsi d'assurer l'exclusivité des circulations de films à la rotation rapide (environ 1000 titres par an) sur ces vastes étendues où le coût de transport des copies (par avion souvent) jouait un rôle non négligeable. Cette intégration de toutes les fonctions dans la diffusion du film était, de facto, indispensable pour assurer la rentabilité, voire la simple alimentation d'un marché limité à seulement trois centaines de salles. Après leur rachat en 1974 par la Sopacia, un consortium conduit par l'UGC française qui s'en délestera dix ans plus tard au vu de sa très faible profitabilité et de l'absence de régulation des marchés par les États, aucune entreprise africaine, privée comme nationalisée, ne sera en mesure, politique comme économique, de jouer ce rôle que les réalités géographiques et démographiques avaient imposées au fil des décennies antérieures.

18 À cette destruction de la distribution durant les années 1980 se juxtaposeront des phénomènes déjà décrits ${ }^{15}$ qui achèveront la destruction de l'étroit marché des salles durant les décennies 1990/2000 : la faiblesse et la précarité des revenus de la majorité des populations; une ruralité prédominante avec dispersion des habitats; l'effet d'une urbanisation croissante attirant une population à très faible pouvoir d'achat ; l'insécurité en de nombreux quartiers de villes ; les conflits armés endémiques sur de larges portions de territoires ; certains codes moraux ou religieux qui peuvent contraindre et interdire de cinéma des catégories de la population, notamment les femmes ; l'impact des télévisions puis de la vidéo, du piratage et d'internet (en ville) ; la fraude, le non-professionnalisme, l'absence d'investissement des exploitants, etc. La chute de l'empire soviétique provoquera la cessation de ses soutiens au début des années 1990, et les plans d'ajustements structurels, imposés par les institutions financières internationales en raison de la dérive des comptes publics des États, précéderont de peu la dévaluation de 50 \% du franc CFA imposée par la France (1994). Cette situation amènera certains États à se désengager totalement du secteur culturel ${ }^{16}$, ces facteurs jouant très souvent le rôle de prétextes commodes pour un personnel politique qui ne s'était en réalité jamais réellement soucié du cinéma.

Quelques actions sporadiques et isolées d'États ont bien eu lieu, telles la création de structures de type CNC (au Cameroun, Burkina Faso, etc.), d'embryon de législation (Mali, Guinée, etc.), l'attribution de budgets dédiés (récemment au Sénégal et en Côte d'Ivoire). Mais les annonces ont été bien supérieures aux réalisations effectives, le manque de constance et d'efficacité reflétant un désintérêt ancien et profond de la quasi-totalité des dirigeants de ces pays, qui n'y ont vu ni intérêt économique (objectivement faible), ni outil potentiel de valorisation symbolique. Le manque de volonté politique de tous les 
gouvernements de l'ensemble des nations concernées a surtout empêché une protection de la filière cinématographique, tout en ne favorisant pas le dynamisme commercial par une régulation adaptée, tel qu'observable en zone anglophone. Juridiquement, cette absence de lois comme de contrôle des entreprises, et la domination de l'économie informelle échappant à la transparence et à l'impôt, s'explicitent notamment par le maintien en place d'élites liées à des clans familiaux et/ou tribaux qui n'ont aucun intérêt au développement, notamment culturel, de leur pays, et dont l'enrichissement personnel et la mauvaise gouvernance sont tolérés tant qu'ils maintiennent une stabilité dont a besoin la France pour la continuité de son approvisionnement en matières premières stratégiques, notamment énergétiques ${ }^{17}$. A contrario l'Angleterre a cessé depuis longtemps d'intervenir, directement ou non, dans les affaires de ses anciennes colonies, privilégiant des accords souples, et une législation propice aux échanges commerciaux et au dynamisme entrepreneurial.

Depuis le début du XXI ${ }^{\mathrm{e}}$ siècle, l'explosion démographique, la croissance économique de certaines villes tendant vers des mégalopoles, ont généré l'embryon d'une classe moyenne, soucieuse de consommations, y compris culturelles, copiées sur celles du reste du monde, dont le modèle envié devient accessible via les télévisions satellitaires étrangères, et internet, de plus en plus répandus. Le groupe français Bolloré, présent antérieurement sur les ports en eaux profondes et les transports, a lancé une offensive inédite sur toute l'ASF après son rachat de Vivendi qui contrôlait Canal+. Outre en 2014 ses nouvelles offres de bouquets financièrement accessibles (abonnements mensuels de 5 000 à $20000 \mathrm{FCFA}^{18}$ ), pour les alimenter la chaîne a lancé en 2016 un plan pluri annuel de plusieurs millions d'euros, destiné à produire, dans chacun des pays, des séries et émissions nationales, afin de répondre à la demande grandissante de toutes les populations de voir, enfin, des programmes avec des sujets et des acteurs qui leur ressemblent. Parallèlement, le groupe a commencé à construire à partir de 2016 dix-sept salles ${ }^{19}$ comprenant une salle mono-écran de cinéma (fermée, gradinée) jouxtant une salle de concert-spectacle (extérieure, découverte), dénommées Canal Olympia, afin de provoquer un fort effet de notoriété lié aux deux marques dont le groupe est propriétaire. $\mathrm{Si}$, deux ans après leur lancement, on peut demeurer dubitatif sur leurs positionnements et pérennité (localisation parfois excentrée, limitée aux capitales, tarifs ciblant la classe moyenne, programmation similaire dans toutes les villes, diffusion de blockbusters étatsuniens et de quelques films nigérians ${ }^{20}$ ), d'autres groupes comme Pathé commencent également à s'implanter ${ }^{21}$, tandis que quelques complexes cinématographiques émergent timidement en quelques villes dynamiques (Abidjan, Dakar..).

21 Après avoir chassé la France de la zone, ce retour d'entreprises françaises dans le cinéma après trente ans d'absence interroge sur l'ampleur et la durée du marasme africain francophone. Nonobstant, compte tenu de l'absence durable d'attention et d'implication des États d'ASF, si tant est qu'il renaisse, il y a encore loin avant la reconstruction d'un marché cinématographique sur cette partie du continent, tant que les conditions réglementaires, juridiques et fiscales ne seront pas remplies. En cela, la situation contraste avec les pays anglophones (Nigeria, Ghana, Ouganda, Kenya...) qui produisent désormais, chacun, davantage de longs métrages que la plupart des pays européens, faisant vivre plusieurs centaines de milliers de personnes, et qui ont vu des multiplexes se construire depuis une décennie, issus des groupes sud-africains, mais aussi nationaux. En ASF plus qu'ailleurs, l'accroissement de la porosité de la frontière historique entre cinéma et audiovisuel l'a totalement diluée, et la rend caduque tant esthétiquement 
qu'économiquement. Un nouveau marché aux contours non encore définis a émergé, selon un modèle de financement et de fonctionnement en voie de profond et rapide réagencement. S'ils ne sont plus économiquement indispensables à la filière, les lieux de projection collectifs et payants demeurent néanmoins nécessaires, à la fois pour valoriser symboliquement et distinguer certaines œuvres, les films, mais aussi pour permettre un certain type de socialisation, de culture, et de distraction. La grande inconnue est ce fait historique inédit: alors qu'une large partie de la population, au moins urbaine, a présentement un accès quantitativement inconnu à des images issues du monde entier, une pleine génération, instruite, curieuse et ouverte sur le monde, n'a jamais mis les pieds dans une salle de cinéma. Si le modèle européo-centré (salles 3D, voire 4DX...) qui est en cours de construction va probablement séduire la fraction minoritaire la plus riche des capitales, quelles salles (et quels films), pour la partie, majoritaire, la plus pauvre de la population, dont, hormis le Burkina Faso durant une brève période, leurs États ne se sont jamais souciés?

\section{BIBLIOGRAPHIE}

Barlet, Cinémas d'Afrique des années 2000, Paris, L'Harmattan, 2012.

Boughédir, Le cinéma africain de A à Z, Bruxelles, OCIC, 1987.

Caillé et Forest (dir.), Regarder des films en Afriques, Villeneuve d'Ascq, Presses universitaires du Septentrion, 2017.

Carrière, Le marché du film dans les nouveaux États de l'Afrique tropicale atlantique, UniFrance Film, 1962.

Dupré, Le FESPACO, une affaire d'État(s) 1969-2009, Paris, L'Harmattan, 2012.

Forest (dir.), L'industrie cinématographique en Afrique, De Boeck, nº 238, Bruxelles, 2011.

Forest, Économie du cinéma en Afrique sud saharienne francophone. Produire (1960-2018), Paris, L'Harmattan, 2018.

Millet « (In) dépendance des cinéma du Sud \&/vs France » dans Creton (dir.) Cinéma et (in) dépendance, Théorème $\mathrm{n}^{\circ} 5$, Presses de la Sorbonne nouvelle, 1998.

Roitfeld, Afrique noire francophone, UniFrance Film, septembre 1980.

Traoré, Le viol de l'imaginaire, Paris, Fayard, 2010.

Vieyra, Le cinéma africain, Des origines à 1973, Paris, Présence Africaine, 1975.

\section{NOTES}

1. VIEYRA Paulin Soumanou, Le cinéma africain, Des origines à 1973, Paris, Présence Africaine, 1975, $444 \mathrm{p}$. 
2. Outre les articles de J.-R. Dèbrix, il faut citer les trois rapports d'Unifrance : Michel Carrière, Le marché du film dans les nouveaux Etats de l'Afrique tropicale atlantique, UniFrance Film, 1962, 274 p. ; M. De Place, Rapport d'enquête sur le cinéma au Sénégal et en Côte d'Ivoire, UniFrance Film, 1970; Pierre Roitfeld, Afrique noire francophone, UniFrance Film, septembre 1980, 90 p., ainsi que l'unique ouvrage de référence pour les premières décennies : Pierre Pommier, Cinéma et développement en Afrique noire francophone, Paris, Pedone, 1974, $188 \mathrm{p}$.

3. Nous utilisons le préfixe « sud » (saharienne) car « sub » signifie "sous » ce qui, à la fois peut relever d'une connotation hiérarchique péjorative, et également se référer à une géographie qui ne renvoie pas au réel physique, mais à une convention de représentation de domination spatiale, imposée par les géographes des puissances coloniales; dans notre système solaire il n'existe ni haut, ni bas.

4. Sur cette question, voir notamment Claude Forest, Économie du cinéma en Afrique sud saharienne francophone. Produire (1960-2018), Paris, L'Harmattan, 2018, p. 65-84.

5. Colin Dupré, Le FESPACO, une affaire d'État(s) 1969-2009, Paris, L'Harmattan, 2012, 406 p.

6. Férid Boughedir, Cinéma africain et décolonisation, Université Sorbonne Nouvelle Paris III, $1976,550 \mathrm{p}$.

7. Tahar Cheriaa, Écrans d'abondance... ou cinéma de libération en Afrique ?, Tunisie-SATPEC/ Libye-El Khayala, 1978, $312 \mathrm{p}$.

8. Nationalisation des salles en Haute Volta dès 1970, puis au Mali et Niger; de la distribution en 1974 au Sénégal, Bénin et Tanzanie, en 1975 Madagascar, en 1979 aux Congo, Somalie et Soudan. Toutes seront des échecs et conduiront à la disparition ou au retour au privé des salles concernées en une décennie.

9. «L'écrasante majorité des films étrangers importés prône la violence, le racisme, et la haine entre les peuples et (...) relève indubitablement de la drogue plutôt que de la culture », Tahar Cheriaa, op. cit., p. 32, «Ces initiatives des trusts procèdent de la pire entreprise de subversion et de déviation culturelle consciente, préméditée, réfléchie et planifiée » ibid., p. 132.

10. Notamment Jean-René Débrix, Situation du cinéma en Afrique francophone, Afrique contemporaine, $\mathrm{n}^{\circ} 40$, Paris, Documentation française, nov.-déc. 1968, p. 2-6.

11. Tahar Cheriaa, Écrans d'abondance... ou cinémas de libération en Afrique, op. cit.

12. Raphael Millet «(In)dépendance des cinéma du Sud \&/vs France » dans Laurent Creton (dir.) Cinéma et (in)dépendance, Théorème n5, Presses de la Sorbonne nouvelle, 1998, p. 160.

13. " (Ils permettraient annuellement) de produire en Afrique 150 à 200 films de long métrage ", Tahar Cheriaa, op. cit., p. 72.

14. À ce jour (2018), il faut noter l'absence d'étude détaillée sur les deux entreprises SecmaComacico, comme sur celle qui les racheta, la Sopacia fililale de l'UGC, pas plus d'ailleurs que sur le CIDC.

15. Claude Forest, L'industrie du cinéma en Afrique, (dir.), Bruxelles, De Boeck, Afrique contemporaine, $\mathrm{n}^{\circ} 238,2011$.

16. Aminata Traoré, Le viol de l'imaginaire, Paris, Fayard, 2010, 206 p.

17. Mali, Niger, Côte d'Ivoire, Centrafrique, Rwanda, etc. l'armée française n'a jamais cessé d'être présente en ASF depuis la naissance du cinéma, d'y faire la guerre, et de tuer des Africains.

18. 7,50 à $30 €$.

19. Huit étaient construites à l'été 2018 : Douala et Yaoundé au Cameroun, Cotonou au Benin, Lomé au Togo, Conakry en Guinée, Dakar au Sénégal, Ouagadougou au Burkina Faso, Niamey au Niger. D'autres étaient en construction : Bamako au Mali, Libreville et Port Gentil au Gabon, Accra au Ghana et Banjul en Gambie, etc. 
20. http://canalolympia.com/

21. La formule du petit multiplexe semble avoir été retenu par le groupe : huit écrans à Tunis en 2018, qui seront suivis par Casablanca où s'implantera la direction «Afrique ", puis Dakar et Abidjan (annoncés pour 2019).

\section{RÉSUMÉS}

Depuis un demi-siècle, la faible production de films issue des pays d'Afrique sud saharienne francophone a été très inégalement distribuée en salles de cinéma, à l'étranger essentiellement en France, mais quasiment pas sur leurs marchés nationaux, faute de lieux en activité. Pourtant, les mouvements post indépendantistes conduits par des réalisateurs africains avaient débouché sur de nombreuses initiatives en direction des production et diffusion du film. La contribution tente d'approfondir l'étude, sur cette longue période qui suit les indépendances, des causes de la destruction de la diffusion en salles en ASF, et de questionner les conditions d'une éventuelle reconstruction pérenne.

For half a century, the low production of movies from the French-speaking South Saharan countries has been very unequally distributed in cinemas, abroad mainly in France, but almost not in their national markets, for lack of places in activies. However, the post-independence movements led by African filmmakers had led to numerous initiatives of movie production and broadcasting. The contribution attempts to deepen the study, over the long period following independence, of the causes of the destruction of distribution in ASF movie theaters, and to question the conditions of a possible long-term reconstruction.

\section{AUTEUR}

\section{CLAUDE FOREST}

Claude Forest est Professeur en Etudes cinématographiques à l'Université de Strasbourg.Il a notamment dirigé les ouvrages suivants :Regarder des films en Afriques (co-dir. avec Patricia Caillé),P. U. du Septentrion, 2017 ;Au cinéma en Afrique, Espaces \& Signes, 2017 ; Les salles de cinéma. Histoire et géographie, Montréal, revue Cinémas, vol. 27, n²-3, printemps 2018 ; Produire des films. Afriques et Moyen-Orient, P. U. du Septentrion, 2018 ; Économie du cinéma en Afrique sud saharienne francophone. Produire (1960-2018), Paris, L'Harmattan, 2018 ; Pratiques spectatorielles en Afriques francophones. Enjeux d'une empirie comparative,(co-dir. avec Patricia Caillé),P. U. du Septentrion, 2019. 